\title{
¿CUÁLES SON LAS ACTITUDES Y CREENCIAS EN TORNO A LA CIENCIA DE LOS PROFESORES Y ESTUDIANTES DE PROFESORADO DE FÍSICA DEL URUGUAY?
}

\author{
What are the attitudes and beliefs about Science of the \\ Physics teachers and future Physics teachers in Uruguay?
}

\author{
Álvaro Suárez \\ ORCID https://orcid.org/0000-0002-5345-5565 \\ Consejo de Formación en Educación, Instituto de Profesores Artigas, Montevideo, Uruguay \\ alsua@,outlook.com

\section{Daniel Baccino} \\ ORCID https://orcid.org/0000-0001-5572-2623 \\ Consejo de Formación en Educación, Instituto de Profesores Artigas, Montevideo, Uruguay \\ dbaccisi@gmail.com
}

\section{Martín Monteiro}

ORCID https://orcid.org/0000-0001-9472-2116

Universidad ORT Uruguay, Montevideo, Uruguay

monteiro@ort.edu.uy

\section{Arturo C. Martí}

ORCID https://orcid.org/0000-0003-2023-8676

Instituto de Física, Facultad de Ciencias, Udelar, Montevideo, Uruguay

marti@fisica.edu.uy

Recibido: 9 de abril de 2021

Aceptado: 9 de noviembre 2021

\section{Resumen:}

Investigamos las concepciones epistemológicas de los profesores y futuros profesores de Física del Uruguay por medio de la aplicación del test CLASS (Colorado Learning Attitudes about Science Survey), uno de los instrumentos más aceptados en la comunidad de investigación en enseñanza de la Física. Los resultados obtenidos permiten comparar las actitudes y creencias en torno a la ciencias de ambos colectivos y evaluar en forma cuantitativa el acuerdo o no con las concepciones de expertos en la materia. Primero presentamos un pantallazo general de las respuestas y luego identificamos categorías en las que existen similitudes o diferencias significativas entre ambos colectivos estudiados y a su vez con la referencia de los expertos. Las categorías que muestran variaciones positivas o negativas significativas entre las opiniones de los profesores y futuros profesores indican las áreas donde la formación es favorable o desfavorable. Por otro lado, las áreas donde las diferencias con las opiniones de los expertos es globalmente notoria nos sugieren que deben ser fortalecidas en todos los ámbitos. Para tener una perspectiva más global, también comparamos nuestros resultados con algunos provistos por la literatura. Finalmente, dejamos una serie de preguntas que pensamos pueden favorecer indagaciones posteriores.

Palabras clave: Epistemología, actitudes y creencias, profesores de Física, formación docente. 


\begin{abstract}
:
We investigate the epistemological conceptions of the uruguayan Physics teachers and future Physics teachers through the application of the CLASS test (Colorado Learning Attitudes about Science Survey), one of the most accepted instruments in the research community in Physics education. The results obtained allow us to compare the attitudes and beliefs about science of both groups and quantitatively evaluate the agreement or not with the conceptions of experts in the field. First, we present a general screenshot of the responses and then we identify categories in which there are significant similarities or differences between the two groups studied and in turn with the reference of the experts. The categories that show significant positive or negative variations between the opinions of future teachers and teachers indicate the areas where training is favorable or unfavorable. On the other hand, the areas where the differences with the opinions of the experts are globally notorious suggest that they should be strengthened. To get a more global perspective, we also compare our results with those published in the literature. Finally, we raise several questions that we think may favor further inquiries.
\end{abstract}

Keywords: Epistemology, attitudes and beliefs, physics teachers, teaching formation.

\title{
1. Introducción
}

El famoso sicólogo estadounidense B. F. Skinner señaló en 1964 que "la educación es aquello que perdura una vez que se olvidó todo lo aprendido.” Esta frase resume en forma ilustrativa que los contenidos específicos que enseñamos son solo una pequeña parte de la transformación que tiene lugar a medida que los estudiantes transitan por el sistema educativo. En efecto, los estudiantes no son "cajas vacias" donde los docentes depositan conocimientos, sino que vienen con toda una carga de conocimientos de la propia materia, una historia personal, una forma de interaccionar con sus compañeros y docentes, un posicionamiento frente al aprendizaje y un conjunto de expectativas de diversa índole entre otros aspectos. A menudo subestimados en relación a los disciplinares, dichos aspectos juegan un papel importante en la transformación que esperamos experimenten los estudiantes.

El conjunto de ideas, suposiciones y concepciones previas sobre la Ciencia, en especial su evolución, sus métodos, su validación o refutación se engloban en el concepto de creencias epistemológicas. Estas creencias, que en general no son explícitas, impactan fuertemente en la forma de enseñar y aprender (Redish, Saul y Steinberg, 1998). Por mencionar algunos aspectos, ¿consideramos la ciencia un conjunto de conocimientos grabados en piedra o un sistema en continua evolución? El rol de la experimentación, ¿es simplemente un requisito más para verificar las teorías o forma parte sustancial a la hora de entender la naturaleza, falsear teorías o delimitar su rango de validez? El posicionamiento frente a estas preguntas no es neutro. Si consideramos los conocimientos previos como inamovibles, no hace falta fomentar una actitud crítica ni interesarse por los nuevos avances. Asimismo, si consideramos que "todo vale" o que la evidencia experimental no es relevante caemos en el campo de las creencias seudocientíficas, o posturas tales como las sostenidas por terraplanistas o antivacunas.

Los docentes, en cada acción u omisión que realizamos en clase, estamos directa o indirectamente incidiendo sobre las actitudes y creencias de nuestros estudiantes en el aprendizaje y la enseñanza de la Física. Pensemos por ejemplo una situación hipotética, de una clase sobre cinemática donde resolvemos un problema en el pizarrón. Imaginemos que, como parte del procedimiento de resolución, escribimos las ecuaciones que describen un MRUV y les decimos a los estudiantes que para hallar la respuesta deben "buscar la ecuación que tenga todos los datos excepto el pedido y simplemente despejar". En una situación hipotética como la descrita, estaríamos sin quererlo, 
dándole una imagen errónea al estudiante de la manera en la se deben resolver los problemas en Física. En ese sentido, estamos formando (sin quererlo) una imagen incorrecta de la ciencia y de cómo aprender Física.

Los aspectos que enmarcan el conocimiento científico propio de la disciplina en la sociedad merecen ser discutidos, analizados y explicitados en las clases de ciencias. Es cierto que si bien la Filosofía como asignatura está presente en la mayoría de planes de estudio, muchos aspectos específicos de su relación con la Física deben ser discutidos junto con sus contenidos específicos. ¿Es posible hablar de inducción electromagnética sin mencionar el papel que juegan los motores y generadores en nuestra vida cotidiana? ¿Podemos presentar la mecánica cuántica como una teoría abstracta mientras sostenemos en la mano un teléfono inteligente con millones de transistores que se rigen justamente por las leyes de la mecánica cuántica? ¿Es razonable acaso estudiar los detalles de la fisión del uranio sin mencionar las implicaciones sociales y éticas de la bomba atómica? Estos ejemplos muestran la estrecha vinculación entre los contenidos que estudiamos y el conjunto de creencias y posturas en torno a la ciencia.

Existen otros aspectos más sutiles, no tan visibles, en torno a las actitudes frente a la ciencia que se discuten aún menos en nuestras clases. Algunos de estos involucran a las actitudes frente minorías o géneros y en especial al papel de las mujeres en relación a las "ciencias duras". ¿Por qué es menor el porcentaje de mujeres que eligen estas disciplinas? ¿La enseñanza que brindamos es "neutra" frente a estos aspectos? ¿En qué momento de la formación se origina esta aparente falta de interés? ¿Ocurre en los primeros años? ¿O en etapas más avanzadas? ¿Se debe a las condiciones labores? ¿Influye la imagen típica del científico que se presenta en la sociedad? ¿Se trata de una discriminación del estilo del "club de Tobi"'? Todas estas preguntas están cobrando mayor importancia hoy día. Para avanzar en la dirección de responder en forma convincente resulta natural indagar en las actitudes y creencias epistemológicas de los estudiantes y de los docentes.

Las actitudes y creencias tienen múltiples dimensiones. Una de ellas es la transversal que recorre los diferentes colectivos desde las posturas de la sociedad, de los estudiantes de ciencias, de los futuros docentes de Física, de los actuales docentes hasta los investigadores de la disciplina. En este trabajo nos enfocamos en evaluar y discutir las actitudes y creencias de los docentes de Física de enseñanza media y de los estudiantes de profesorado de Física en Uruguay. A continuación, en la próxima sección exponemos un conjunto de herramientas desarrolladas en los últimos años para indagar estos aspectos que constituyen nuestro marco teórico y presentamos los resultados más relevantes que se han obtenido de su aplicación en diversos contextos. Avanzando en los aspectos centrales de este trabajo, concernientes a las actitudes y creencias de los docentes uruguayos, en la sección 3, presentamos la metodología empleada y en la sección 4 los principales resultados. Finalmente, la discusión y consideraciones finales se expone en la sección 5.

1 Expresión tomada de la popular historieta "La pequeña Lulu" y utilizada en América Latina para 


\section{Herramientas de análisis de las creencias epistemológicas y sus principales resultados}

2.1. Cuestionarios estandarizados en la investigación en Enseñanza de la Física

En diferentes ámbitos de la Física y de su enseñanza se comenzó a observar desde hace casi medio siglo que frecuentemente los estudiantes aprendían a resolver los problemas y aprobaban sus cursos, pero, al enfrentarse a problemas conceptuales sencillos, mostraban no tener una comprensión cabal de los fenómenos estudiados (Viennot, 1979; Trowbridge y McDermott, 1980; Halloun y Hestenes, 1985; Hake, 1996, Docktor y Mestre, 2014). A partir de estas observaciones, surgieron voces desde el núcleo de la Física que reclamaban la necesidad de cambios. A lo largo de los años siguientes los estudios en esta temática fueron adquiriendo coherencia surgiendo un nuevo campo con identidad propia: la investigación en enseñanza de la Física (PER por sus siglas en inglés) (McDermott, 1999) que apunta a generar conocimiento basado en métodos científicos ampliamente aceptados y en especial en contar con resultados cuantitativos con respaldo estadístico sólido (Docktor y Mestre, 2014).

Uno de los pilares de la PER es la evaluación sistemática de conocimientos. En este marco se idearon diversos cuestionarios para la evaluación de conocimientos destacándose el Force Concept Inventory (Hestenes, Wells y Swackhamer, 1992). Este cuestionario, aplicado a miles de estudiantes en cientos de universidades, apunta a evaluar el pasaje entre una concepción aristotélica de la mecánica a una newtoniana. La principal conclusión derivada del análisis de los resultados es que los métodos de enseñanza activa, en los que los estudiantes se involucran activamente, redundan en mejores aprendizajes en comparación con los métodos tradicionales, principalmente clases magistrales, donde el estudiante tiene una actitud pasiva. Esta mayor "ganancia" en el aprendizaje, es un resultado robusto en términos estadísticos, no depende del estudiante, del docente ni del contexto donde ocurre el aprendizaje (Hake, 1998).

En los años posteriores se propusieron numerosos cuestionarios, como el Conceptual Survey of Electricity and Magnetism (CSEM), Malonney et al. (2001) o el Brief electricity and magnetism assesssment (BEMA), Chabay y Sherwood (2006), que apuntan a otros campos de la Física. Si bien en los comienzos la elaboración de estos cuestionarios era relativamente artesanal, con el pasar del tiempo se definieron una serie de pasos, requisitos estadísticos y etapas que debe cumplir cada propuesta para ser aceptada en la comunidad (Heron y Meltzer, 2005). Paralelamente, en varios sitios colaborativos (el más reconocido es http://www.physport.org) se publican los cuestionarios, generalmente de libre acceso, con enlaces a los artículos, información complementaria como traducciones a muchos idiomas, planillas de cálculo y otras herramientas para facilitar su implementación. Por otro, cuando se cumplen estos requisitos es posible compartir la información, tanto subir nuestros resultados como acceder a los de otras instituciones.

\subsection{El análisis de las actitudes y creencias epistemológicas}

El campo de las actitudes y creencias epistemológicas no escapa a la pretensión de los investigadores de obtener información cuantitativa. En ese sentido, se han desarrollado en los últimos veinticinco años una serie de cuestionarios o pruebas estandarizadas para evaluar las actitudes y creencias epistemológicas de los estudiantes sobre la Física, su enseñanza y aprendizaje. A diferencia de los test de co- 
nocimientos específicos, basados mayoritariamente en preguntas múltiple opción, los test de actitudes y creencias suelen proponer a los estudiantes que indiquen su grado de acuerdo o desacuerdo (conocido como escala de Likert) con afirmaciones que reflejan (o no) la opinión de "expertos" de la disciplina (es decir, físicos profesionales, investigadores). Cada afirmación tiene un valor esperado que es asignado durante el proceso de diseño y validación del instrumento, en base a repetidas interacciones con expertos. Las diferencias entre las respuestas de los estudiantes y las respuestas de los expertos, constituye la materia prima para analizar el estado epistemológico del grupo de estudiantes. En general es de interés analizar el impacto que determinados cursos y enfoques de enseñanza tienen en las actitudes y creencias de los estudiantes. Con este fin se aplica la prueba al menos dos veces, la primera al comenzar el curso (pretest) y la segunda al terminarlo (postest). La diferencia entre ambas pruebas permite medir los cambios en las actitudes y creencias en función de elementos demográficos, metodologías y estrategias de enseñanza.

Dentro del conjunto de pruebas estandarizadas se destacan especialmente tres: el MPEX (Maryland Physics Expectations Survey) (Redish, et al., 1998), que como su nombre lo indica, apunta a sondear las expectativas de los estudiantes en relación a la Física, el CLASS (Colorado Learning Attitudes about Science Survey) (Adams, et al., 2006), que apunta a evaluar las creencias de los estudiantes sobre la Física y sobre su aprendizaje y el E-CLASS (Colorado Learning Attitudes about Science Survey for Experimental Physics) (Wilcox y Lewandowski, 2016) que sigue la misma orientación del CLASS pero apunta a los aspectos experimentales de Física.

\subsection{Métodos de enseñanza y posturas epistemológicas}

En la literatura de PER se han publicado numerosos trabajos indagando en la relación entre los métodos y resultados de la enseñanza y las posturas epistemológicas de los estudiantes (Madsen, McKagan y Sayre, 2015). En particular se ha mostrado que ciertas posturas epistemológicas "negativas" de los estudiantes son sin duda un obstáculo para el desarrollo de una educación científica de calidad pues afectan la manera en que los alumnos aprenden y abordan los cursos de ciencias (Perkins, et al., 2005; Milner-Bolotin, Antimirova, Noack y Petrov, 2011). Por ejemplo, las maneras que un estudiante cree que debe aprender Física, sus prácticas metacognitivas, así como la imagen que tiene de la Física como ciencia, son algunos de los aspectos del currículum oculto que deberían afectar la forma de aprender. Un estudiante que cree que la Física consiste principalmente en hechos y fórmulas desconectadas, estudiará de manera diferente a otro que la ve como una red de conceptos interconectados. Aquellos que ven el conocimiento de la Física como una red coherente de ideas, tienen motivos para realizar prácticas metacognitivas para monitorear su aprendizaje (Redish, Saul y Steinberg, 1998). En relación con el rendimiento académico, diversos estudios han mostrado la existencia de una correlación positiva con las puntuaciones del pretest del CLASS y el MPEX (Perkins, et al., 2005; Cahill, et al., 2018). En los últimos años, se han realizado múltiples investigaciones cuantitativas sobre los cambios que se producen en las actitudes y creencias de los estudiantes en función de su formación previa, tipos de cursos y estrategias de enseñanza utilizadas, así como la relación entre el pretest y el rendimiento académico, entre otras variables (Madsen, McKagan y Sayre, 2015). 
Del conjunto de investigaciones, queremos destacar particularmente aquellas que estudian los cambios en las posturas epistemológicas en función de los métodos de enseñanza, así como las que analizan la relación entre dichas posturas y el entendimiento conceptual. A partir de un meta-análisis realizado examinando 24 trabajos de investigación publicados, Madsen, McKagan y Sayre (2015) clasificaron las estrategias de enseñanza de los cursos de Física en tres categorías en función de los cambios en las posturas epistemológicas de los estudiantes:

A) Cursos donde se produce un deterioro de las actitudes y creencias de los estudiantes (el resultado promedio del postest sobre actitudes y creencias es menor al del pretest). En esta categoría se encuentran los cursos enfocados con metodologías de enseñanza tradicionales, así como aquellos desarrollados con algunas de las metodologías de enseñanza activa más difundidas, tales como la "Instrucción de pares" y "Tutoriales de Física Introductoria" (Adams et al, 2006; Madsen, et al., 2015). Aunque el hecho de que un curso tradicional no promueva las actitudes y creencias adecuadas podría ser algo previsible, a priori resulta llamativo que, para cursos basados en algunas estrategias de enseñanza activas, los estudiantes también tengan un desempeño menor en el CLASS y el MPEX después de haber transitado por el curso.

B) Cursos donde no se detecta una diferencia significativa en las actitudes y creencias de los estudiantes antes y después del curso. Aquí se encuentran los cursos donde se toman en cuenta algunos aspectos epistemológicos en su implementación (Kohl y Vincent Kuo, 2012; Madsen, et al., 2015). En estos cursos se implementan estrategias de enseñanza que promueven el desarrollo de habilidades de razonamiento, la realización de inferencias a partir de las observaciones y reflexiones sobre por qué confiamos en las ideas científicas, entre otras.

C) Cursos donde se detecta una mejora significativa en las actitudes y creencias de los estudiantes. En este grupo se encuentran dos tipos: por un lado, aquellos que son radicalmente reestructurados, basados en la construcción de modelos (Brewe, et al., 2013) y por otro, los que tienen un fuerte énfasis en aspectos epistemológicos (Elby, 2001; Redish y Hammer, 2009). En los cursos basados en la construcción de modelos, los estudiantes trabajan en pequeños grupos, realizan experimentos y analizan los resultados con el fin de elaborar modelos de los diferentes fenómenos. Un aspecto central de este tipo de estrategias es la puesta en común de las conclusiones de cada equipo y la promoción de discusiones con otros compañeros de forma de permitir a los estudiantes validar y refinar los modelos construidos. Por otro lado, en los cursos con un fuerte énfasis en lo epistemológico, se desarrollan actividades donde se promueve la metacognición, y se trabaja por ejemplo con tutoriales que enfatizan la reconciliación entre las ideas intuitivas y pensamiento científico formal.

Vemos entonces que, como se desprende de la literatura, exceptuando aquellos cursos pensados específicamente para promover mejoras en las actitudes y creencias de los estudiantes, en general los alumnos las deterioran como efecto colateral de los cursos de Física. Sorpresivamente, al finalizar muchos de los cursos de Física, más alumnos creen por ejemplo que la Física está menos conectada con el mundo real, es menos coherente, el razonamiento es menos importante y el aprendizaje memorístico resulta útil. Estos resultados, a priori sumamente chocantes, nos interpelan sobre la promoción en forma involuntaria de actitudes y creencias contrarias a las que 
esperaríamos se desarrollaran de manera natural por el solo tránsito a través de los cursos. La estrecha relación entre posturas epistemológicas y resultados del proceso educativo justifica ampliamente la necesidad de conocer y actuar sobre las actitudes y creencias de los estudiantes a lo largo de todo el sistema educativo.

\section{Metodología}

En la investigación que presentamos en este trabajo optamos por utilizar CLASS, la encuesta de actitudes de aprendizaje de la ciencia desarrollada por el grupo de PER de la Universidad de Colorado Boulder, en Estados Unidos (Adams, et al., 2006). Este cuestionario cuenta con muy amplia aceptación dado que ha pasado un exhaustivo proceso de validación. Para su creación se partió de herramientas como el MPEX y VASS para luego revisarlo en base a entrevistas con estudiantes y expertos (16 físicos con amplia experiencia en la enseñanza que estuvieron de acuerdo con las respuestas para casi todas las preguntas). Las categorías de CLASS se crearon utilizando un análisis factorial de base reducida, donde las categorías estadísticas en bruto y las categorías predeterminadas por los investigadores se combinaron de forma iterativa. Finalmente, fue aplicado a miles de estudiantes y se verificó que aquellos con más experiencia en física, tenían creencias más parecidas a las de los expertos. Los resultados de su aplicación en diferentes contextos geográficos y sociales o en grupos donde se aplican diferentes estrategias de enseñanza se encuentran disponibles para su análisis y comparación (Milner-Bolotin, Antimirova, Noack y Petrov, 2011; Ding, 2013; Suwonjandee, Mahachok y Asavapibhop, 2018; Balta, Cessna, Kaliyeva, 2020, Kontro y Buschhüter, 2020; Nissen et al., 2021). Este instrumento valora las actitudes de los estudiantes para aprender Física, cómo piensan que se relaciona la Física con su vida cotidiana y qué piensan sobre la Física. Está conformado por 42 afirmaciones que se presentan cada una con escala de Likert de cinco niveles, desde completamente en desacuerdo (1), hasta completamente de acuerdo (5). Anotamos algunas preguntas a modo de ejemplo:

- "Yo pienso en la Física que se involucra en mi vida cotidiana."

- "El conocimiento en Física consiste de muchos temas desconectados."

- "Después de estudiar un tema de Física y siento que lo entiendo, tengo dificultades para resolver problemas sobre el mismo tema"

- "Cuando resuelvo un problema de Física, busco una ecuación que utiliza las variables dadas en el problema y substituyo los valores"

Las afirmaciones se pueden organizar, según los autores de CLASS, en ocho categorías (de forma no exclusiva), tal como se muestra en la tabla 1. Al procesar una encuesta realizada por un estudiante, cada una de sus respuestas recibirá un valor (-1 o +1), según el grado de desacuerdo $(-1)$ o acuerdo $(+1)$ entre la respuesta dada por el estudiante y la respuesta típica de los expertos. Este criterio se aplica solamente a las 36 preguntas en las que existe real consensos entre los expertos (solo 27 de las 36 están categorizadas). Por el contrario, no se aplica a las seis preguntas (ver la última fila de la tabla 1) que no muestran acuerdo entre expertos. A partir de estas valoraciones se determinan los niveles de acuerdo ("favorable" y "desfavorable") para cada una de las ocho categorías definidas por los autores, así como para la encuesta en su totalidad. Este proceso se aplica a todos los individuos del grupo analizado y finalmente se calculan los promedios para cada afirmación, categoría y el total de la encuesta. 
Tabla 1. Identificación de categorías y las declaraciones comprendidas en cada una de ellas (Traducción de Adams et al, 2006, de los autores)

\begin{tabular}{|l|c|c|}
\hline \multicolumn{1}{|c|}{ Categoría } & Afirmaciones & ID \\
\hline Conexión con el mundo real (Real world conection) & $28,30,35,37$ & RWC \\
\hline Interés personal (Personal interest) & $3,11,14,25,28,30$ & PI \\
\hline Hacer sentido / esfuerzo (Sense making effort) & $11,23,24,32,36,39,42$ & SM/E \\
\hline Entendimiento conceptual (Conceptual comprehension) & $1,5,6,13,21,32$ & CC \\
\hline $\begin{array}{l}\text { Entendimiento conceptual aplicado (Applied conceptual } \\
\text { understanding) }\end{array}$ & $1,5,6,8,21,22,40$ s & ACU \\
\hline $\begin{array}{l}\text { Resolución de problemas. General (Problem solving, } \\
\text { general) }\end{array}$ & $13,15,16,25,26,34,40,42$ & PSG \\
\hline $\begin{array}{l}\text { Resolución de problemas. Confianza (Problem solving, } \\
\text { confidence) }\end{array}$ & $15,16,34,40$ & PSC \\
\hline $\begin{array}{l}\text { Resolución de problemas. Sofisticación (Problem solving } \\
\text { sophistication) }\end{array}$ & $5,21,22,25,34,40$ & PSS \\
\hline \begin{tabular}{l} 
Sin acuerdo entre expertos \\
\hline
\end{tabular} & $4,7,9,31,33,41$ & \\
\hline
\end{tabular}

La propuesta del test se implementó a través un formulario electrónico, enviado mediante convocatorias abiertas, a profesores de Física con actividad en educación media, y estudiantes de primer año del profesorado de Física del CFE (Consejo de Formación en Educación de Uruguay). La encuesta estuvo abierta para cada colectivo, docentes y estudiantes de todo Uruguay, durante un mes en la primera mitad del año 2020.

Recibimos 143 respuestas de profsesores. El género declarado por los encuestados fue: Mujer (46\% del total), Hombre (52 \%), no lo declaró un $2 \%$. El $52 \%$ de los encuestados indica que desarrolla su actividad docente en Montevideo (la capital del país), mientras que el $48 \%$ restante se distribuye en otros departamentos del país. En cuanto a la edad de los encuestados, $31 \%$ declara entre 31 y 40 años, $22 \%$ se declaran en las franjas etarias contiguas a la anterior (18-30 y 41-50), $25 \%$ registra más de 51 años de edad. ${ }^{2}$

Un total de 138 estudiantes de casi todos los centros en los que se imparte el profesorado de Física contestaron la encuesta. Con respecto al género, $62 \%$ de los encuestados declaran ser mujer, 38 \% declara ser hombre, y ningún encuestado tomó las otras opciones disponibles. En términos del Instituto donde están radicados, la mayoría de ellos (56 \%) cursa en la modalidad semipresencial ${ }^{3}$, mientras que el resto lo hace en forma presencial en diferentes centros del país.

Los resultados de cada una de las encuestas se analizaron en base a las categorías definidas y validadas por los autores del instrumento. Para cuantificar los resultados, con ese punto de partida, utilizamos una planilla electrónica desarrollada por los autores (extraída del portal de Physport) y adaptada por los autores de este trabajo. En la sección siguiente mostramos los principales resultados obtenidos.

2 Se puede acceder a los resultados de la encuesta a profesores y estudiantes a través de los siguientes enlaces: bit.ly/CLASS-profes-UY2020 y bit.ly/CLASS-formacion-UY2020

3 Semipresencial consiste en una modalidad de cursado de la carrera de profesorado, habilitada (desde el año 2003) para los Centros de Formación Docente de todo el Uruguay, que no cuenten en su oferta con las asignaturas específicas en algunas Especialidades, entre las que cuenta el profesorado de Física. 


\section{Análisis de los resultados}

\subsection{Profesores y estudiantes de profesorado del Uruguay}

En un primer análisis de los resultados, damos una mirada a todas las respuestas categorizadas de los profesores. En el histograma de la figura 1 se representan las frecuencias (cantidad de profesores) cuyas respuestas se encuentran de acuerdo con los expertos, en intervalos de ancho 10 puntos porcentuales. Por ejemplo, un total de 56 profesores dieron respuestas "favorables" que están entre 80 \% y 90 \%. La forma típica de caracterizar a esa distribución es mediante el promedio, que en este caso es $80 \%$, la desviación estándar (13\%) y la desviación estándar del promedio (1\%).

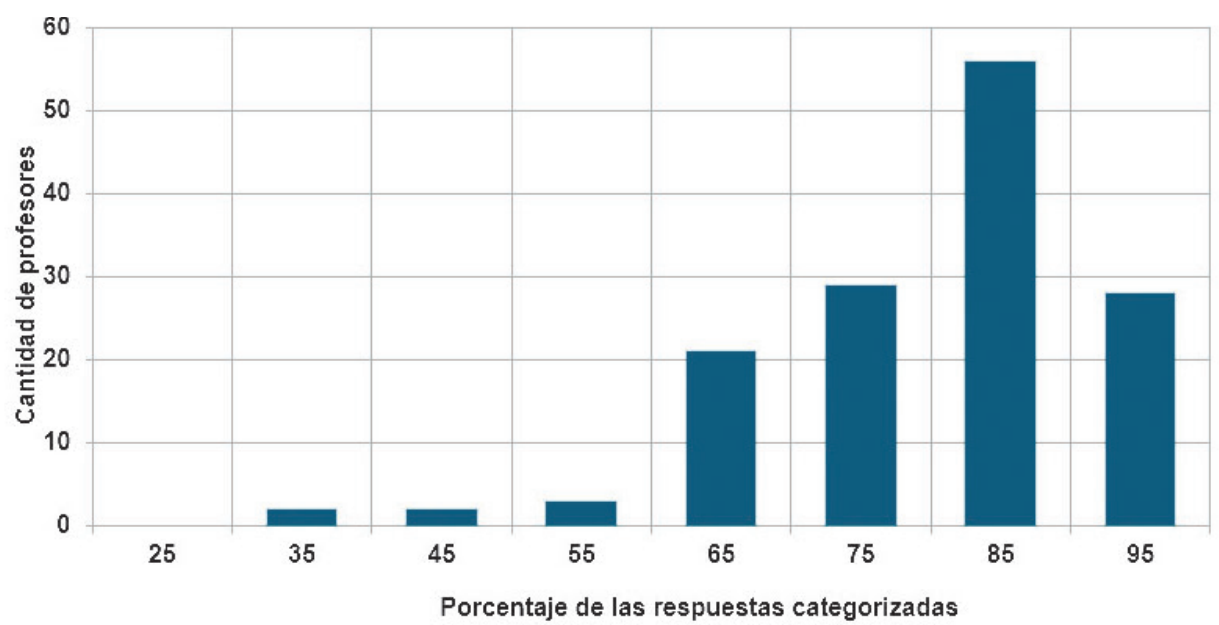

Figura 1. Resultados globales de las respuestas a las preguntas categorizadas en el test CLASS por parte de 143 profesores de Física de Uruguay. Fuente: elaboración propia.

En la figura 2 mostramos los resultados por categorías en la encuesta a profesores, para respuestas favorables, neutras y no favorables (según el ID de la tabla 1); las dos primeras columnas representan los porcentajes correspondientes a todas las respuestas (All) y al total de respuestas categorizadas (All C.) respectivamente.

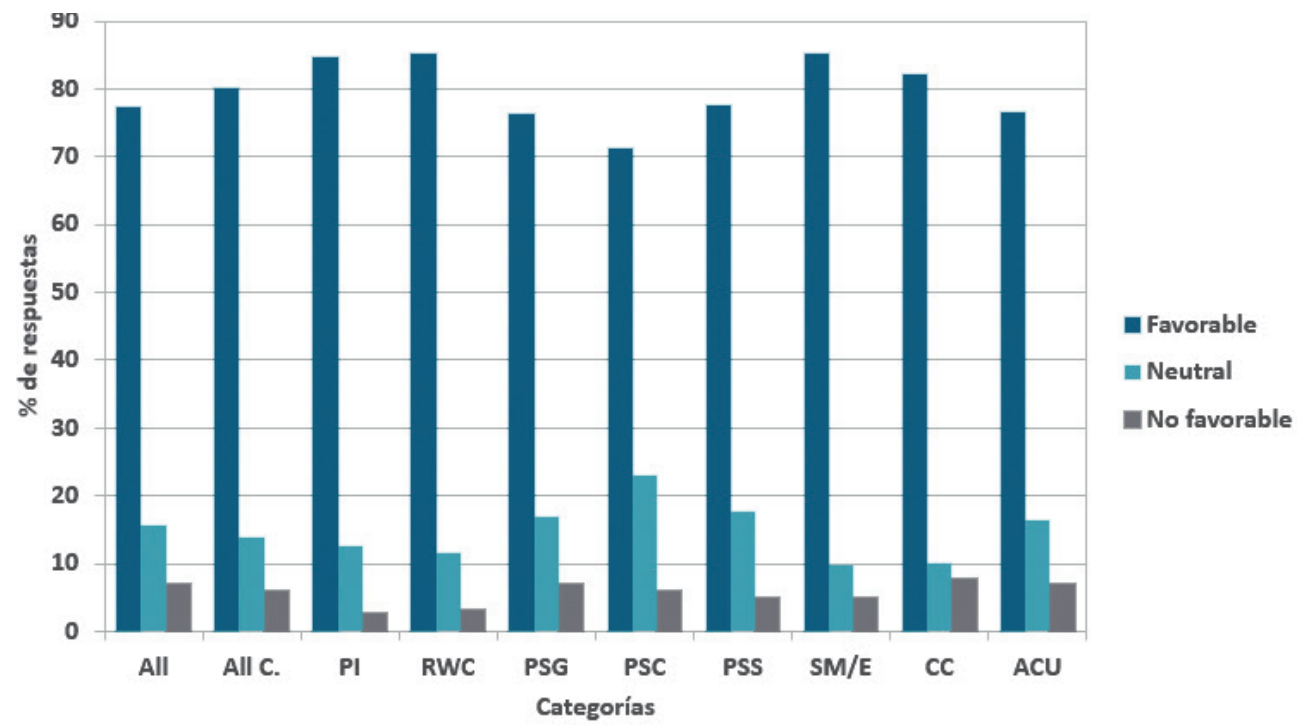

Figura 2. Desempeño de los profesores de Física encuestados con el test CLASS desglosado por categorías. Fuente: elaboración propia. 
El gráfico de la figura 3 muestra los resultados de respuestas favorables de los profesores y de los estudiantes de profesorado de Física discriminado por categorías de CLASS (corresponden idénticos comentarios sobre la identificación de categorías que las realizadas sobre el gráfico de la figura 2). Se representa, para cada categoría y en ambos grupos, el error estándar del promedio. El eje de porcentajes se define entre valores de $45 \%$ y $95 \%$, con el objetivo de visualizar mejor la zona de interés.

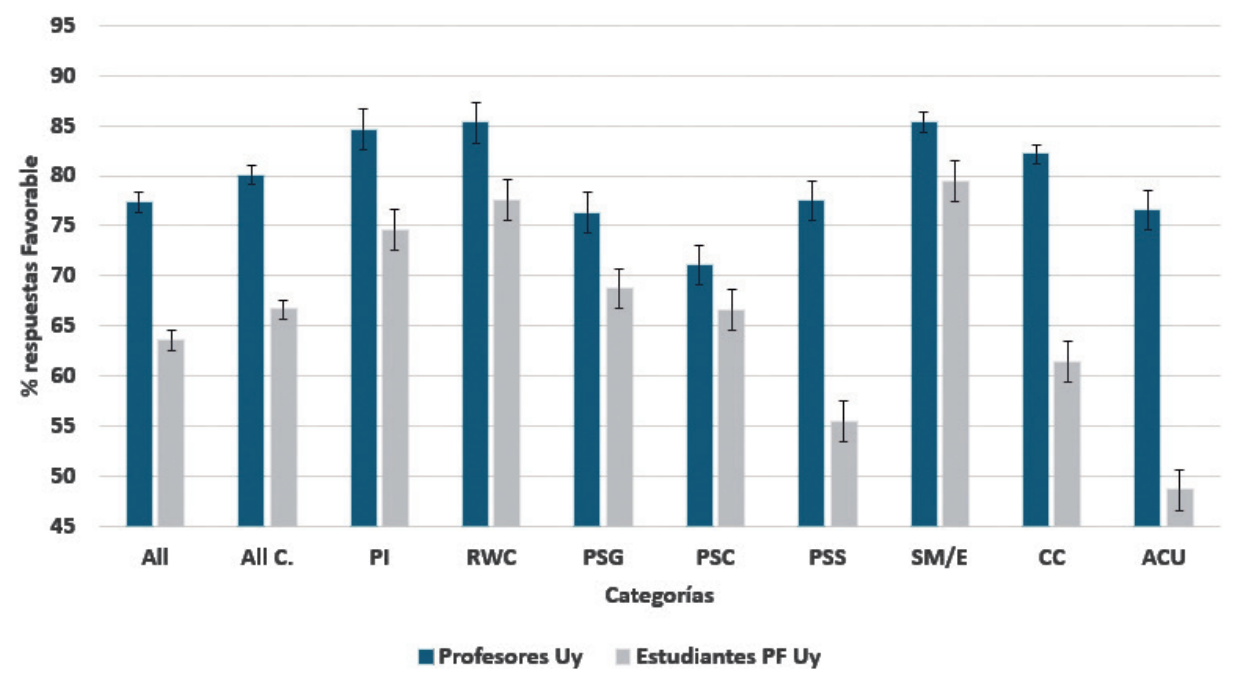

Figura 3. Desempeño de 138 estudiantes de profesorado de Física (Estudiantes PF Uy) y 143 profesores de Física en actividad (Profesores Uy) en el test CLASS desglosado por categoría. Fuente: elaboración propia

Un aspecto que resalta a la vista en el gráfico es la diferencia en los promedios de las respuestas de profesores y estudiantes. En el caso de profesores el promedio de respuestas categorizadas es $80 \%$, mientras que en estudiantes es $67 \%$. Si consideramos el rango definido por los promedios de todas las categorías, observamos que en los estudiantes es de aproximadamente 30 puntos porcentuales, mientras que en caso de los profesores es aproximadamente 15 puntos. En el caso de los profesores las respuestas a las categorías están menos dispersas que en los subconjuntos categorizados para los estudiantes.

Las categorías en las que hay más acuerdo con los expertos son compartidas por estudiantes y profesores. En algunas categorías el acuerdo de estudiantes y profesores con expertos es muy similar, por ejemplo, en Esfuerzo/Hacer sentido y Confianza en la Resolución de problemas. En el otro extremo, donde se visualizan diferencias mayores, se encuentran las categorías correspondientes al Entendimiento conceptual aplicado y Sofisticación en la resolución de problemas. Asumiendo que es posible considerar a los dos grupos de individuos encuestados como parte de una mirada pseudo-longitudinal, una hipótesis que puede explicar estas diferencias, refiere a los procesos de formación sistemática y los mediados por la práctica cotidiana de los docentes.

Propusimos la encuesta a 79 estudiantes que ingresaron a las Licenciaturas en Física y Matemática (Facultad de Ciencias, Udelar) y a 105 estudiantes que ingresaron a la Facultad de Ingeniería (Udelar). No encontramos diferencias significativas entre las respuestas de los estudiantes universitarios y los estudiantes de profesorado de Física encuestados. La misma encuesta se propuso a investigadores locales en el área de las Ciencias Físicas. Al comparar estos últimos resultados con los de los profeso- 
res de física, no encontramos diferencias significativas. Los resultados del análisis de las respuestas de estos colectivos serán analizados en otro trabajo.

\subsection{Comparación con resultados internacionales}

Es interesante comparar los resultados globales de los estudiantes de profesorado de Física de Uruguay, con estudiantes universitarios de otras partes del mundo. Utilizando datos del meta-análisis realizado por Madsen, McKagan y Sayre (2015) y de Bates, Galloway, Loptson y Slaughter (2011), tomamos los valores promedio de la implementación del test CLASS a estudiantes de trece universidades de Canadá, Estados Unidos y el Reino Unido, con la particularidad que el perfil de las carreras de dichos estudiantes es tal que toman cursos de Física basados en cálculo. Este punto es de particular importancia, ya que según la literatura (Madsen, McKagan y Sayre, 2015), los estudiantes que realizan carreras con fuerte énfasis en Física y Matemática, obtienen mejores resultados en el CLASS.

Mientras que el promedio del test CLASS a los estudiantes de formación docente para las 36 preguntas donde hay acuerdo entre expertos fue de 63,5 \%, el promedio para los trece grupos es de 64,1\% con una desviación estándar de 6,0 \%. Estos resultados, muestran cómo los estudiantes del profesorado de Física de Uruguay, obtienen resultados similares a las poblaciones utilizadas para la comparación. Aunque no nos dicen nada respecto a los sistemas educativos, sugieren que quizás haya una tendencia en los resultados promedio del test CLASS para estudiantes con perfiles hacia el área de ciencias e ingeniería, independientemente de su país de origen.

Por otra parte, en relación a las actitudes y creencias de los profesores de física, puede resultar constructiva la comparación con un estudio realizado en Tailandia, un país con características demográficas, sociales, económicas y culturales muy diferentes a las de Uruguay. En el estudio realizado por Suwonjandee, Mahachok y Asavapibhop (2018), se analizaron las respuestas de 196 profesores de física y 211 estudiantes de secundaria. A pesar de las diferencias mencionadas entre los contextos de ambos colectivos docentes, las respuestas fueron muy similares en la mayoría de las preguntas y categorías del CLASS. Solamente en dos de las 42 preguntas del test se evidencian diferencias significativas entre los profesores de Tailandia y de Uruguay (preguntas 8 y 22). Estas son dos de las seis preguntas de la categoría Entendimiento conceptual aplicado.

En las preguntas mencionadas, los profesores de Uruguay mostraron un mayor grado de acuerdo con los expertos, que los profesores de Tailandia. Algo muy similar a lo que ocurre con los estudiantes de profesorado de física de Uruguay, que también muestran grandes diferencias con los expertos precisamente en estas mismas dos preguntas. De hecho, puede ser relevante señalar que estas preguntas son las que en nuestra investigación presentan el mayor grado de discrepancia entre las respuestas de los profesores de física y los estudiantes de profesorado de física:

- Pregunta 8: "Cuando resuelvo un problema de física, busco una ecuación que utiliza las variables dadas en el problema y sustituyo los valores." El grado de acuerdo con los expertos fue de $60 \%$, $24 \%$ y $9 \%$, para profesores de Uruguay, estudiantes de profesorado de física de Uruguay y profesores de Tailandia, respectivamente.

- Pregunta 22: "Si quiero aplicar un método usado en un problema de física en otro problema, estos problemas deben involucrar situaciones muy similares." El grado de acuerdo con los expertos fue de $67 \%$, $27 \%$ y $38 \%$, para profesores de Uruguay, estudiantes de profesorado de física de Uruguay y profesores de Tailandia, respectivamente. 
Estas dos preguntas parecen señalar que la actitud frente a los problemas de física es un aspecto clave en la formación de los profesores de física y parecen ser una fortaleza relativa de los profesores uruguayos. El bajo grado de acuerdo que los estudiantes de profesorado de física de Uruguay muestran en la pregunta 8, parece sugerir que abordan los problemas de física conectando ecuaciones, resolviéndolas con los datos disponibles, sin pensar demasiado en los aspectos conceptuales. La pregunta 22 parece reforzar esta idea, que los estudiantes de profesorado no logran comprender plenamente los aspectos conceptuales y los principios básicos de la física, y en su lugar aprenden física memorizando ecuaciones, lo cual les impide abordar nuevas situaciones con la confianza necesaria para resolverlas.

\section{Comentarios finales}

Las actitudes y creencias epistemológicas de los profesores y estudiantes juegan un rol fundamental en el aula, involucrando aspectos muy disímiles. En ese sentido, ser conscientes de su importancia y reconocer el impacto que pueden tener nuestras acciones en el aula en la imagen de ciencia que promovemos, la elección de carreras futuras o los logros académicos de nuestros estudiantes, resulta imprescindible.

Las creencias "epistemológicas" de los estudiantes, sus puntos de vista sobre la naturaleza del conocimiento y el aprendizaje, son un obstáculo para el desarrollo de una educación científica de calidad, afectando la manera en que aprenden y abordan los cursos de ciencias. Por ejemplo, quienes consideran que el aprendizaje consiste principalmente en absorber información, tendrán una postura diferente frente al conocimiento que aquellos que consideran que se basa en la comprensión (Elby, 2001). Por ello, una de las claves para mejorar los aprendizajes de nuestros estudiantes es promover posturas epistemológicas adecuadas. Ayudar a los estudiantes, por ejemplo, a comprender la importancia de la consistencia y coherencia del conocimiento, así como la diferencia entre la memorización y una comprensión más profunda, es sin duda un objetivo de enseñanza en sí mismo, como parte del desarrollo de una educación científica de calidad. Reconocer como docentes, las imágenes de ciencia que transmitimos, su relación con la tecnología y la sociedad, cómo evolucionar hacia concepciones que favorezcan el aprendizaje de nuestros estudiantes, sus formas de trabajar y las perspectivas de género, son aspectos fundamentales que debemos tener en cuenta y abordar explícitamente en nuestras clases.

En este trabajo reportamos los resultados obtenidos de la implementación de CLASS a un conjunto importante de profesores y futuros profesores de Física de enseñanza media de Uruguay. El puntaje global de los test muestra que el resultado de los profesores es más alto y con menos dispersión entre categorías que los de los futuros profesores que tienen un valor medio menor y además una mayor dispersión. Otro aspecto importante es que en ambos conjuntos las categorías que tienen mayor acuerdo con la opinión de los expertos son coincidentes, en particular en las de Esfuerzo/Hacer sentido, Conexión con el mundo real e Interés personal. Por otro lado, en las categorías de Entendimiento conceptual aplicado y Sofisticación en la resolución de problemas constatamos las mayores diferencias. Estas observaciones sugieren que estos últimos aspectos son adquiridos durante la formación y práctica profesional. En el extremo opuesto se encuentra la categoría Confianza en la resolución de problemas, donde no hemos encontrado diferencia significativa entre las respuestas de los grupos de profesores $(71 \%)$ y estudiantes de profesorado $(67 \%)$. Estos porcentajes se 
encuentran entre los más bajos (en el caso de los profesores es el más bajo de todas las categorías) para ambos grupos, en relación al promedio respectivo.

Observamos también que la comparación del grupo de estudiantes de profesorado con el grupo de estudiantes que optan por carreras con énfasis en Física y Matemática, presentan puntajes similares. Aunque los resultados de estos grupos de estudiantes son en general buenos, se encuentran claramente por debajo de los presentados por profesores e investigadores. De esta manera, parece clara la necesidad de apostar en enseñanza media a trabajar sobre cuestiones vinculadas a las actitudes y creencias epistemológicas de nuestros estudiantes.

Finalmente, el trabajo realizado deja varias preguntas abiertas por responder en futuras investigaciones. ¿En qué etapa los estudiantes de profesorado transforman sus creencias epistemológicas, acercándose a la de expertos? ¿De qué manera estas creencias impactan en la formación docente? ¿Cómo se pueden repensar los cursos de profesorado y de enseñanza media para impactar de manera positiva en las posturas epistemológicas de los estudiantes? ¿Existen diferencias entre las creencias de los estudiantes del profesorado de Física con las de otras especialidades? Si queremos tener una educación científica de mayor calidad, éstas y otras preguntas relevantes, deben ser investigadas.

\section{Agradecimientos}

Este trabajo fue realizado gracias al apoyo financiero brindado por la ANII y el CFE a través del proyecto "Conociendo e incidiendo sobre las concepciones epistemológicas de los futuros profesores de Física” (FSED_3_2019_1_157320) enmarcado en el programa CFE Investiga. Agrademos a todos los participantes por su apoyo desinteresado.

\section{Referencias}

Adams, W. K., Perkins, K. K., Podolefsky, N. S., Dubson, M., Finkelstein, N. D., \& Wieman, C. E. (2006). New instrument for measuring student beliefs about physics and learning physics: The Colorado Learning Attitudes about Science Survey. Physical review special topics-physics education research, 2(1), 010101.

Balta, N., Cessna, S. G., \& Kaliyeva, A. (2020). Surveying Kazakh high school students' attitudes and beliefs about physics and learning with the Colorado learning attitudes about science survey. Physics Education, 55(6), 065019.

Bates, S. P., Galloway, R. K., Loptson, C., \& Slaughter, K. A. (2011). How attitudes and beliefs about physics change from high school to faculty. Physical Review Special Topics-Physics Education Research, 7(2), 020114.

Brewe, E., Traxler, A., De La Garza, J., \& Kramer, L. H. (2013). Extending positive CLASS results across multiple instructors and multiple classes of Modeling Instruction. Physical Review Special Topics-Physics Education Research, 9(2), 020116.

Cahill, M. J., McDaniel, M. A., Frey, R. F., Hynes, K. M., Repice, M., Zhao, J., \& Trousil, R. (2018). Understanding the relationship between student attitudes and student learning. Physical Review Physics Education Research, 14(1), 010107.

Chabay, R. \& Sherwood, B. (2006). Brief electricity and magnetism assessment. Physical Review Special Topics-Physics Education Research, 2(1), 7-13. 
Ding, L. (2013, January). A comparative study of middle school and high school students' views about physics and learning physics. In AIP Conference Proceedings (Vol. 1513, No. 1, pp. 118-121). American Institute of Physics.

Docktor, J. L., \& Mestre, J. P. (2014). Synthesis of discipline-based education research in physics. Physical Review Special Topics-Physics Education Research, 10(2), 020119.

Elby, A. (2001). Helping physics students learn how to learn. American Journal of Physics, 69(S1), S54-S64.

Hake, R. R. (1998). Interactive-engagement versus traditional methods: A sixthousand-student survey of mechanics test data for introductory physics courses. American journal of Physics, 66(1), 64-74.

Halloun, I. A., \& Hestenes, D. (1985). The initial knowledge state of college physics students. American journal of Physics, 53(11), 1043-1055.

Heron, P. R., \& Meltzer, D. E. (2005). The future of physics education research: Intellectual challenges and practical concerns.

Hestenes, D., Wells, M., \& Swackhamer, G. (1992). Force concept inventory. The physics teacher, 30(3), 141-158.

Kohl, P. B., \& Vincent Kuo, H. (2012). Chronicling a successful secondary implementation of Studio Physics. American Journal of Physics, 80(9), 832-839.

Kontro, I., \& Buschhüter, D. (2020). Validity of Colorado Learning Attitudes about Science Survey for a high-achieving, Finnish population. Physical Review Physics Education Research, 16(2), 020104.

Madsen, A., McKagan, S. B., \& Sayre, E. C. (2015). How physics instruction impacts students' beliefs about learning physics: A meta-analysis of 24 studies. Physical Review Special Topics-Physics Education Research, 11(1), 010115.

Maloney, D. P., O’Kuma, T. L., Hieggelke, C. J., \& Van Heuvelen, A. (2001). Surveying students' conceptual knowledge of electricity and magnetism. American Journal of Physics, 69(S1), S12-S23.

Milner-Bolotin, M., Antimirova, T., Noack, A., \& Petrov, A. (2011). Attitudes about science and conceptual physics learning in university introductory physics courses. Physical Review Special Topics-Physics Education Research, 7(2), 020107.

McDermott, L. C., \& Redish, E. F. (1999). Resource letter: PER-1: Physics education research. American journal of physics, 67(9), 755-767.

Nissen, J. M., Horses, I. H. M., Van Dusen, B., Jariwala, M., \& Close, E. W. (2021). Tools for identifying courses that support development of expertlike physics attitudes. Physical Review Physics Education Research, 17(1), 013103.

Perkins, K. K., Adams, W. K., Pollock, S. J., Finkelstein, N. D., \& Wieman, C. E. (2005, September). Correlating student beliefs with student learning using the Colorado Learning Attitudes about Science Survey. In AIP Conference Proceedings (Vol. 790, No. 1, pp. 61-64). American Institute of Physics.

Redish, E. F., Saul, J. M., \& Steinberg, R. N. (1998). Student expectations in introductory physics. American journal of physics, 66(3), 212-224.

Redish, E. F., \& Hammer, D. (2009). Reinventing college physics for biologists: Explicating an epistemological curriculum. American Journal of Physics, 77(7), 629-642.

Suwonjandee, N., Mahachok, T. \& Asavapibhop, B. (2018). Evaluation of Thai students and teacher's attitudes in physics using Colorado Learning Attitudes about Science Survey (CLASS). IOP Conf. Series: Journal of Physics: Conf. Series 1144, 012124 
Trowbridge, D. E., \& McDermott, L. C. (1980). Investigation of student understanding of the concept of velocity in one dimension. American journal of Physics, 48(12), 1020-1028.

Viennot, L. (1979). Spontaneous reasoning in elementary dynamics. European journal of science education, 1(2), 205-221.

Wilcox, B. R., \& Lewandowski, H. J. (2016). Students' epistemologies about experimental physics: Validating the Colorado Learning Attitudes about Science Survey for experimental physics. Physical Review Physics Education Research, 12(1), 010123. 